\title{
VARIATION DES BESOINS ALIMENTAIRES DES PORCS, EN FONCTION DE LA SAISON
}

\author{
PAR \\ André M. LEROY \\ Professenr de Zootechnie à l'Institut National Agronomique.
}

\section{Etude statistique des résultats observés à la Station expérimentale de Bois- Corbon.}

Croissance moyenne des animaux et indices moyens de consommation aux divers âges.

Influence de la saison sur l'indice de consommation ; étude des causes probables des variations observées.

Importance économique de ces variations.

Conclusion.

L'étude des résultats obtenus à la Station de Bois-Corbon sur des porcs de race Large White engraissés à partir de $25 \mathrm{~kg}$ jusqu'à Ioo $\mathrm{kg}$ dans les conditions habituelles des épreuves de contrôle de consommation et de rendement nous ont permis de faire d'intéressantes observations au sujet de la variation des indices de consommation avec différents facteurs. Parmi ces facteurs, il convient de distinguer ceux qui dépendent des individus examinés - âge, origine, état de santé des sujets - et ceux qui dépendent de l'environnement - température et état hygrométrique de l'atmosphère de la porcherie, ventilation, etc... Il est possible, dans ce premier travail, d'examiner les influences de quelques-uns de ces derniers.

Rappelons que les porcs de Bois-Corbon, logés à raison de trois par loge, sont alimentés avec des farines composées dont les formules ont fait l'objet d'une étude patiente. Les rations de farine sont pesées exactement chaque jour, en trois repas égaux, distribuées à sec, puis mélangées immédiatement avant leur consommation avec environ trois fois leur poids d'eau. Les animaux sont pesés régulièrement chaque semaine, ce qui permet de comparer les gains de poids vif hebdomadaires et les consommations d'aliments correspondantes. D'après la composition des aliments, examinée périodiquement au laboratoire, on en déduit la consommation exprimée en unités fourragères par kilogramme de gain de poids vif, de laquelle dépend directement le coût d'engraissement des animaux considérés. 


\section{I. - Courbe moyenne de croissance des animaux}

Au moyen de l'ensemble des résultats de pesées, nous avons d'abord tracé la courbe de variation de poids vif de l'animal en fonction de l'âge (fig. I), puisque tous les sujets examinés provenaient de portées identifiées dès leur naissance.

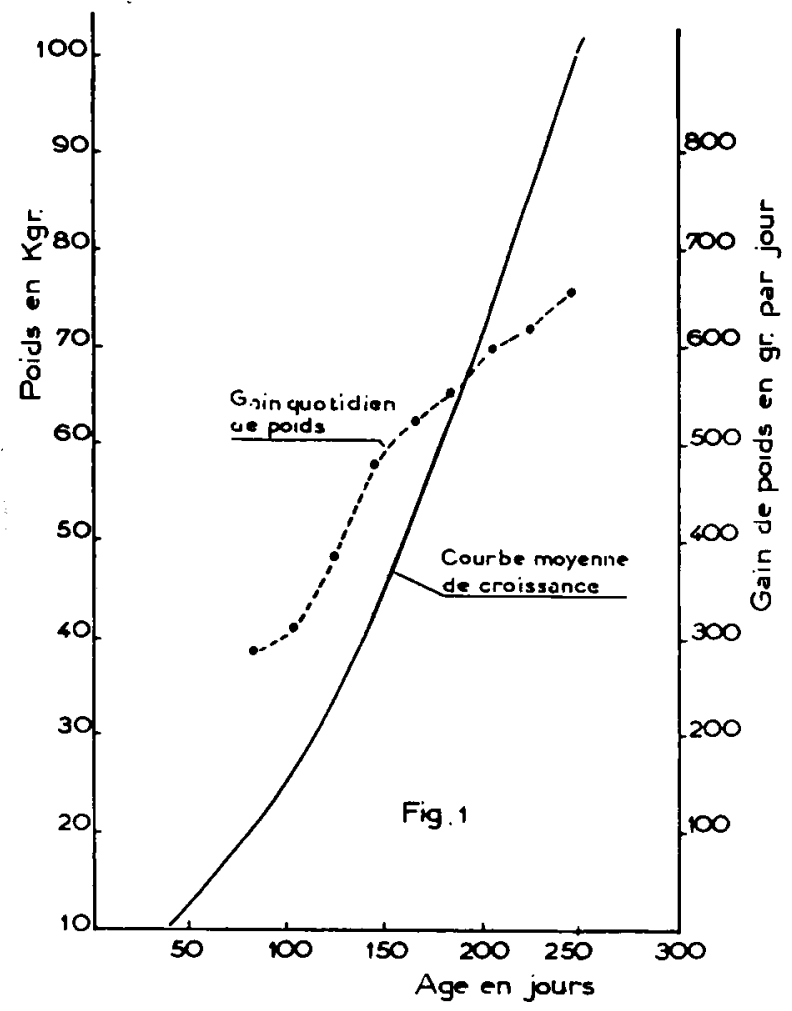

Avec l'aide de ce document, il est possible de calculer le gain moyen quotidien correspondant à chaque âge. Ces données fondamentales figurent sur le tableau ci-après.

\section{TABLEAU I}

Variations des poids et des gains journaliers de poids vit en fonction de l'âge

\begin{tabular}{ccc}
$\begin{array}{cc}\text { Age } \\
\text { en jours. }\end{array}$ & $\begin{array}{c}\text { Poids } \\
\text { correspondant } \\
\mathrm{kg}\end{array}$ & $\begin{array}{c}\text { Gain } \\
\text { quotidien }\end{array}$ \\
\hline 80 & $-\frac{\mathrm{g}}{20}$ & $\mathbf{2 9 0}$ \\
100 & 25,6 & $3 \mathrm{I} 5$ \\
120 & 33,4 & 385 \\
$\mathrm{I} 40$ & 42,0 & 480 \\
$\mathrm{I} 60$ & 51,0 & 525 \\
180 & 61,5 & 555 \\
200 & 74,0 & 600 \\
220 & 87,5 & 620 \\
240 & 98,5 & 660
\end{tabular}




\section{II. - Indice de consommation}

Après avoir groupé les indices de consommation d'après le poids des porcs, nous avons pu suivre la progression de l'indice moyen avec les progrès de la croissance, et constaté l'importante variation des données figurant dans chaque catégorie, reproduite graphiquement dans la figure 2. Voici le tableau de ces indices.

\section{TABLEAU II}

Indices de consommation en fonction du poids des porcs

$$
\begin{aligned}
& \text { Limite des poids. } \\
& \text { Indice correspondant au poids moyen } \\
& \begin{array}{l}
25-35 \mathrm{~kg} \\
35=45= \\
45=55= \\
55=65= \\
65=75= \\
75=85= \\
85-95=
\end{array} \\
& 3,71 \div 0,087 \\
& 3,8 \mathrm{I} \pm 0,074 \\
& 3,89 \pm 0,066 \\
& 4, \mathrm{I} 5 \pm 0,0,084 \\
& 4,30 \pm 0,072 \\
& 4,48 \pm 0,077
\end{aligned}
$$

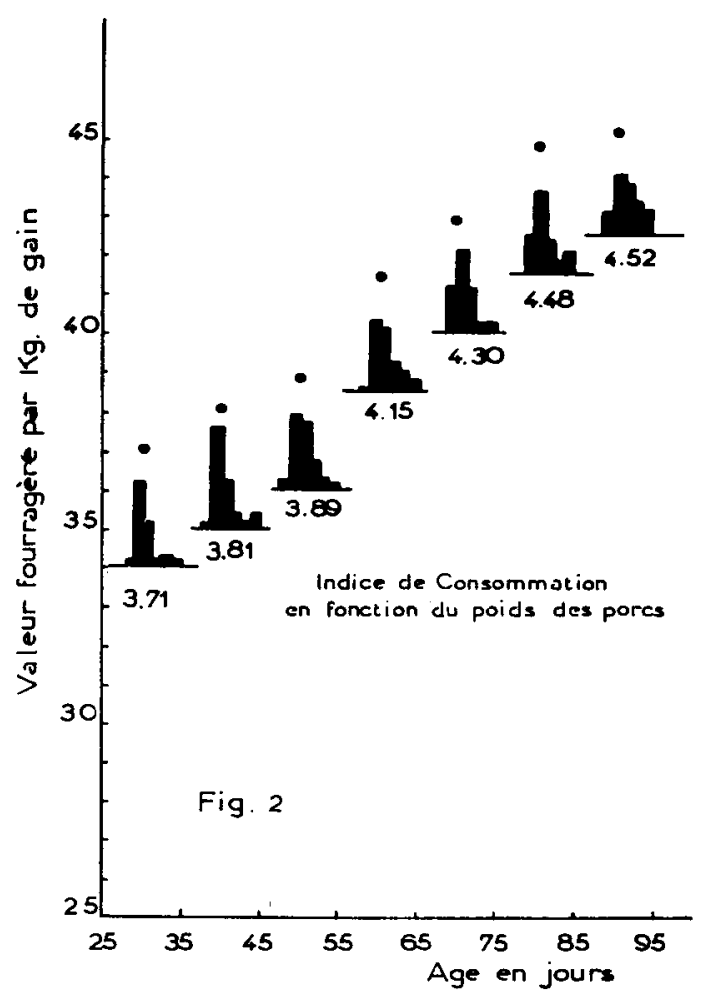




\section{III. - Influence de la saison sur l'indice de consommation}

En nous référant aux dates d'observation, nous pouvons pour chaque catégorie de poids prévue au tableau précédent classer à part les données observées au cours de chaque saison, et calculer les moyennes correspondant à ces classements.

Nous obtenons ainsi les résultats du tableau III, qui nous montrent l'existence d'importantes variations saisonnières, d'intensité et parfois de sens différents, pour les catégories de poids que nous avons adoptées.

\section{TABLEAU III}

\section{Influence des saisons sur l'indice de consommation}

\begin{tabular}{|c|c|c|c|c|}
\hline Limites des poids. & Hiver. & Printemps. & Été. & Automne. \\
\hline $25-35 \mathrm{~kg}$ & $3,64 \pm 0,17$ & $3,66 \pm 0,20$ & $3,9 \circ \pm 0,20$ & $3,85 \div 0,14$ \\
\hline $35-45-$ & $4,37 \pm 0,20$ & $3,69 \pm 0,16$ & $3,38 \pm 0,12$ & $3,62 \pm 0$, II \\
\hline $45-55-$ & $4,26 \pm 0, \mathrm{II}$ & $3,94 \pm 0,17$ & $3,18 \mp 0,09$ & $3,90 \pm 0,12$ \\
\hline $55-65-$ & $4,82 \pm 0,20$ & $4,07 \pm 0,18$ & $3,64 \pm 0,19$ & $3,92 \pm 0,10$ \\
\hline $65-75-$ & $4,3^{2} \pm 0,12$ & $4,29 \pm 0,13$ & $4,25 \pm 0,2 \mathrm{I}$ & $3,94 \pm 0,09$ \\
\hline $75-85-$ & $4,47 \pm 0,13$ & $4,73 \pm 0,17$ & $4,25 \pm 0,19$ & $4,42 \pm 0,13$ \\
\hline $\begin{array}{c}85-95- \\
\text { on par rapport }\end{array}$ & $4,5^{2} \mp 0, \mathrm{II}$ & $4,78 \pm 0,1 \mathrm{I}$ & $4,6 \mathrm{I} \pm 0,19$ & $4,50 \pm 0,12$ \\
\hline supposée égale à roo. & 103,9 & 101, 6 & 96,3 & 97,7 \\
\hline
\end{tabular}

L'examen du tableau montre que l'été et l'automne sont des saisons plus favorables à l'engraissement que le printemps et surtout que l'hiver. Mais cette influence du microclimat environnant ne s'exerce pas de la même façon pour toutes les catégories d'âge. Pour la classe des plus jeunes animaux, l'influence de l'été semble particulièrement défavorable, contrairement à ce que l'on observe pour toutes les autres classes. D'autre part, l'économie de gain observée à la belle saison est beaucoup plus sensible pour les animaux de 35 à $65 \mathrm{~kg}$ que pour les porcs ayant dépassé ce dernier poids, vraisemblablement parce que les fortes températures de certains jours d'été gênent dans une certaine mesure l'organisme des sujets qui se trouvent à ce moment dans un état avancé d'engraissement. En fait, ce sont les animaux des catégories intermédiaires qui paraissent de beaucoup les plus sensibles aux influences saisonnières.

I orsque l'on examine le détail des données d'observation, il est assez fréquent de constater pour certaines périodes de courte durée des anomalies de croissance, qui entraînent passagèrement l'apparition d'indices de consommation exagérément élevés. En considérant comme anormal tout indice de consommation supérieur ou égal à 6 , nous avons pu relever le pourcentage d'observations de cette nature, correspondant aux différentes saisons, et les résultats de notre recherche figurent dans le nouveau tableau ci-après : 


\section{TABLEAU IV}

Influence de la saison sur l'apparition de troubles temporaires de l'indice de consommation

Répartition pour Ioo observations des constatations d'indices de consommatiou anormaux supérieurs à 6 .

Animaux de moins de $55 \mathrm{~kg}$

$\ldots$

Hiver. Printemps.

Été. Automne.

Animaux de plus de
Tous animaux réunis.

39,2
$3 \mathrm{I}, 6$

$3 \mathrm{I}, 6$

$\begin{array}{lr}\overline{\mathrm{I}} 7,4 & \overline{8,6} \\ 28,9 & 26,3\end{array}$

8,6
26,3

34,8

34,4

24,6

I 9,7

$\mathrm{I} 3,2$

$2 \mathrm{I}, 3$

Il est incontestable, d'après ce document, que l'état sanitaire des animaux est dans une certaine mesure responsable des variations de l'indice. Les catégories de porcs pesant moins de $55 \mathrm{~kg}$ sont plus sensibles à l'influence de la saison froide que celles des animaux à la fois plus lourds et plus âgés.

Mais, pour avoir une connaissance plus approfondie de ces influences, du climat, il est utile de calculer pour chaque mois le gain moyen de poids vif pour l'ensemble des porcs présents dans la porcherie, ainsi que l'indice de consommation moyen correspondant. Les données recueillies, qui figurent sur le tableau $\mathrm{V}$ et ont servi à la construction des graphiques de la figure 3 , correspondent au cas d'un animal d'un poids de $60 \mathrm{~kg}$.

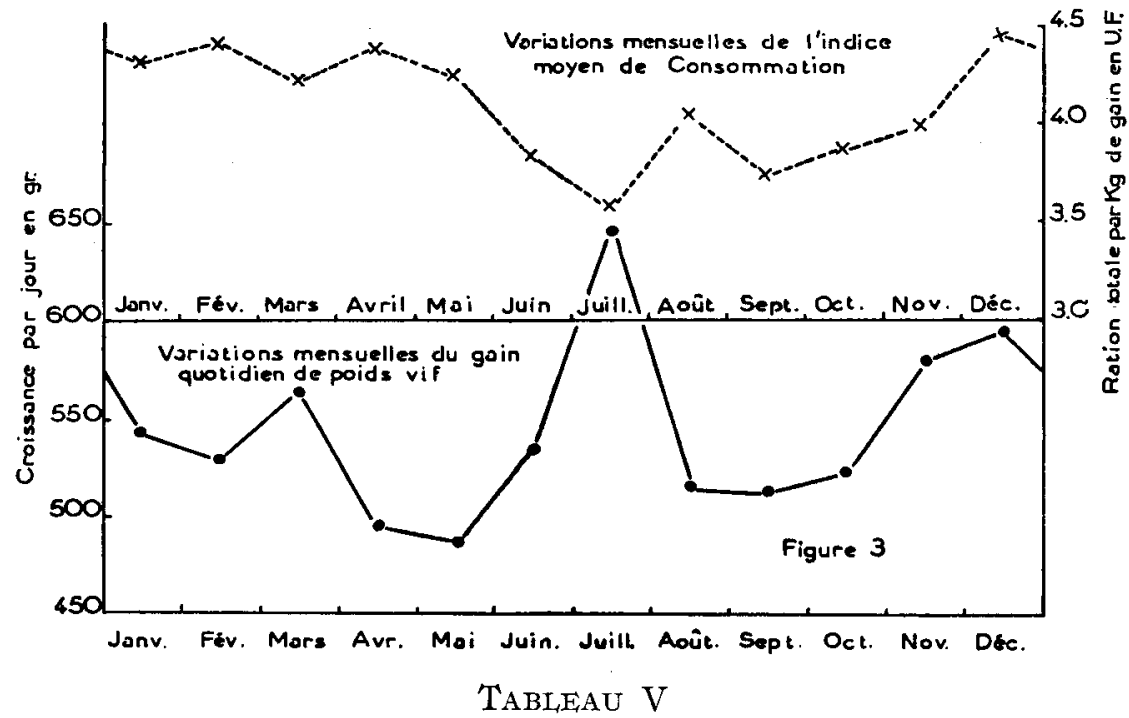

Variations mensuelles du gain quotidien moyen de poids vif et de l'indice moyen de consommation, correspondant à un porc de $60 \mathrm{~kg}$.

\begin{tabular}{|c|c|c|c|c|c|c|}
\hline & Janv. & Févr. & Mars & Avr. & Mai & Juin \\
\hline Gain en $g \ldots \ldots \ldots \ldots \ldots \ldots$. & 545 & 530 & 566 & 496 & 488 & \\
\hline Indice de consommation $\ldots \ldots \ldots \ldots \ldots \ldots$ & 4,33 & $4,4 \mathrm{I}$ & 4,24 & 4,40 & 4,26 & \\
\hline & Juil. & Août & Sept. & Oct. & Nov. & Déc. \\
\hline Gain en & 649 & 515 & $5 \mathrm{II}$ & $5^{2 \mathrm{I}}$ & 580 & \\
\hline Indice de consommation ............ & 3,57 & 4,06 & $\varepsilon_{74}$ & 3,87 & 3,99 & \\
\hline
\end{tabular}


On voit aussi que le gain de poids vif décroît irrégulièrement de décembre à mai, puis remonte très rapidement de mai à juillet. Après une nouvelle baisse pendant les mois d'août et septembre correspondant à des périodes particulièrement chaudes, une nouvelle augmentation s'est produite à la fin de l'année. L'examen du tableau montre, d'autre part, qu'il n'existe pas de corrélation importante entre les gains journaliers de poids vif et les indices de consommation correspondants (Indice de corrélation $r=-0,24)$.

\section{IV. - Influence de la saison sur la ration de production par $\mathrm{kg}$ de gain, déduction faite des besoins de l'entretien}

Nous savons que pour calculer les besoins énergétiques d'un porc dont on connaît le poids, le gain de poids vif et l'âge, il faut ajouter à sa ration d'entretien $E$ une ration de production $\mathrm{P}$ qui dépend de la composition du gain de poids vif et de la grandeur de ce gain par jour.

Or, pour un porc de $60 \mathrm{~kg}$, la valeur de la ration d'entretien est de o,70 u. f. par jour. A l'aide des données du tableau $\mathrm{V}$, nous pouvons étudier la variation mensuelle de la ration de production par $\mathrm{kg}$ de gain (tableau VI).

\section{Tableau VI}

Variations mensuelles de la ration de production par $k g$ de gain

\begin{tabular}{|c|c|c|c|c|}
\hline Mois & $\begin{array}{l}\text { Consommation } \\
\text { journalière } \\
\text { d'un porc. }\end{array}$ & $\begin{array}{c}\text { Besoin } \\
\text { d'entretien. }\end{array}$ & $\begin{array}{l}\text { Besoin de } \\
\text { production. }\end{array}$ & $\begin{array}{c}\text { Besoin de } \\
\text { production, } \\
\text { par kg de gain. }\end{array}$ \\
\hline 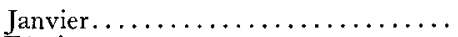 & 2,36 & $0,7 \circ$ & $\mathbf{x}, 66$ & 3,05 \\
\hline Février $\ldots \ldots \ldots \ldots \ldots \ldots \ldots \ldots$ & 2,34 & 0,70 & 1,64 & 3,10 \\
\hline $\operatorname{Mars} \ldots \ldots \ldots \ldots \ldots \ldots \ldots \ldots \ldots$ & 2,40 & 0,70 & $1,7 \circ$ & 3,00 \\
\hline Avril.......... & 2,18 & 0,70 & $I, 48$ & 2,98 \\
\hline 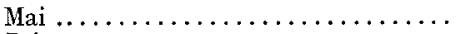 & 2,08 & 0,70 & 1,38 & 2,83 \\
\hline Juin $\ldots \ldots \ldots \ldots \ldots \ldots \ldots \ldots \ldots$ & 2,06 & 0,70 & 1,36 & 2,55 \\
\hline Juillet $\ldots \ldots \ldots \ldots \ldots \ldots \ldots \ldots \ldots$ & 2,52 & 0,70 & $\mathrm{I}, 62$ & 2,50 \\
\hline Août $, \ldots \ldots \ldots, \ldots, \ldots, \ldots \ldots \ldots$ & 2,09 & 0,70 & I,39 & 2,70 \\
\hline Septembre $\ldots \ldots \ldots \ldots \ldots \ldots \ldots \ldots$ & $1,9 \mathrm{I}$ & 0,70 & $\mathrm{I}, 2 \mathrm{I}$ & 2,37 \\
\hline Octobre $\ldots \ldots \ldots \ldots \ldots \ldots \ldots \ldots \ldots$ & 2,02 & 0,70 & 1,32 & 2,54 \\
\hline Novembre $\ldots \ldots \ldots \ldots \ldots \ldots \ldots \ldots \ldots$ & 2,32 & 0,70 & 1,62 & 2,77 \\
\hline Décembre $\ldots \ldots \ldots \ldots \ldots \ldots \ldots \ldots$ & 2,21 & 0,70 & $\mathrm{I}, 5 \mathrm{I}$ & $3, \circ 5$ \\
\hline Période de décembre à mai $\ldots . \ldots \ldots \ldots$ & & $\ldots \ldots \ldots$ & ... & 3,00 \\
\hline ériode de juin à novembre $\ldots \ldots \ldots$ & & & & 2,57 \\
\hline
\end{tabular}

Nous voyons ainsi l'influence considérable exercée par la période froide de l'année sur la dépense énergétique correspondant à l'acquisition d'un kilogramme de croît. Parmi les causes de cet état de choses, il convient de ne pas oublier la différence de composition du gain de poids vif qui se produit sous l'action des basses températures. On sait, en effet, que la formation de graisse à la périphérie du corps est pour le porc une conséquence de la lutte contre le froid. Pour cette raison, les porcs ayant dépassé roo $\mathrm{kg}$ sont considérés par les charcutiers comme ayant trop de 
graisse lorsqu'ils sont abattus en hiver, tandis que les animaux de même poids sont de meilleure qualité pendant l'été. Tandis que le dépôt d'un gramme de matière azotée correspond à un gain énergétique de 5,55 cal., celui d'un gramme de matière grasse exige une dépense de 9,2 cal. Il faut donc, pour obtenir le même poids de tissus de croissance, dépenser d'autant plus d'énergie que les dépôts de graisse tendent à l'emporter davantage sur les matières azotées formées pendant le même temps.

Afin de compléter les renseignements fournis par le tableau VI, nous avons recherché quelles étaient les variations saisonnières de la ration de production, pendant la durée de la période totale d'engraissement, période au cours de laquelle le poids des animaux passe de 25 à Ioo $\mathrm{kg}$. Voici les résultats que nous avons obtenus.

\section{TABLEAU VII}

Influence de la saison sur la ration de production, exprimée en unités fourragères par $\mathrm{kg}$ de gain de poids vif

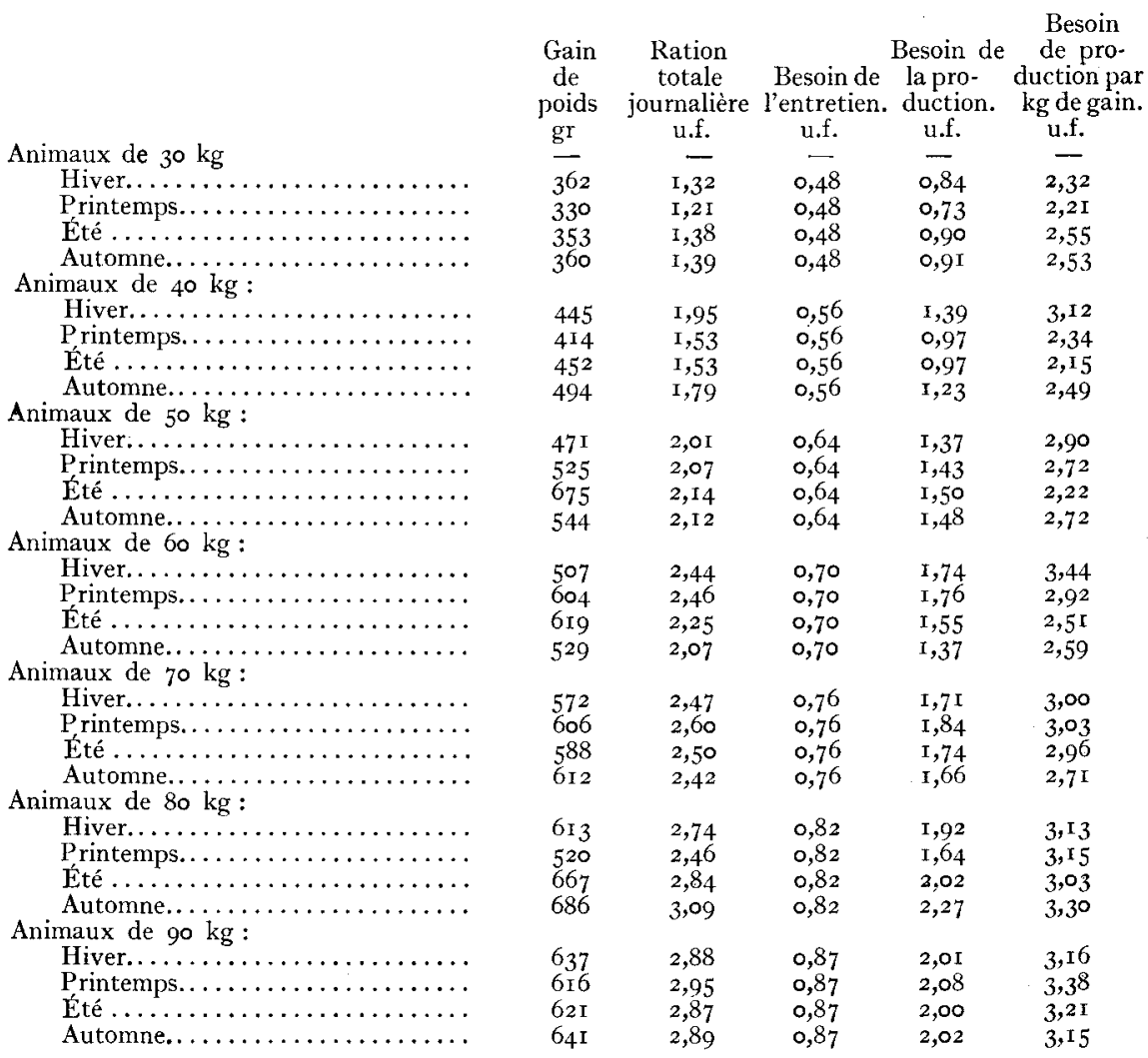

L'utilisation des normes courantes d'alimentation donne donc, dans la pratique, des prévisions qui peuvent s'écarter légèrement dans un sens 
ou dans l'autre, des résultats d'observation. D'une porcherie à une autre, il peut exister des différences dans la croissance des animaux, même si ces porcheries sont garnies d'animaux de même race, de même origine, soumis à la même alimentation distribuée d'une manière identique.

Cette question pose donc le problème de l'influence du microclimat de la porcherie sur la croissance du porc, lequel devrait faire l'objet d'observations systématiques, en raison de son incidence sur la quantité des aliments consommés par $\mathrm{kg}$ de gain.

Pour montrer toute l'importance économique de cette question, nous avons calculé, à l'aide des données du tableau IX, quelle somme totale d'unités fourragères serait nécessaire à un porc qui se trouverait, aux diverses périodes de sa croissance, dans les meilleures conditions possibles, c'est-à-dire dans celles qui correspondent pour chaque catégorie de poids à la ration de production par $\mathrm{kg}$ de gain la plus faible. Nous avons également effectué des calculs analogues, mais en supposant cette fois que nos animaux se trouvent toujours dans les conditions correspondant à la plus forte dépense.

Voici ce que nous avons trouvé :

\section{TABLEAU VIII}

Evaluation de la consommation d'unités fourragères nécessaires pour qu'un porc passe de 25 à $95 \mathrm{~kg}$

\section{I. - Conditions les plus favorables}

\begin{tabular}{|c|c|c|c|c|c|c|}
\hline & & Période. & $\begin{array}{l}\text { Gain } \\
\text { pendant } \\
\text { la période }\end{array}$ & $\begin{array}{l}\text { Durée de } \\
\text { la période. } \\
-\end{array}$ & $\begin{array}{l}\text { Consomm } \\
\text { par } \\
\text { jour. } \\
-\end{array}$ & $\begin{array}{l}\text { Consom- } \\
\text { mation } \\
\text { pour la période }\end{array}$ \\
\hline & & & gr & jours & u.f. & u.f. \\
\hline Poids & bassal & 25 a $35 \mathrm{~kg} \ldots \ldots \ldots \ldots \ldots$ & $33^{\circ}$ & 30,3 & $\mathrm{I}, 2 \mathrm{I}$ & $3^{6,8}$ \\
\hline - & - & 35 à $45-\ldots \ldots \ldots \ldots \ldots$ & $45^{2}$ & $22, \mathrm{I}$ & $\mathrm{I}, 53$ & 34,0 \\
\hline - & - & 45 à $55-\ldots \ldots \ldots \ldots \ldots$ & 675 & $\mathbf{1} 4,8$ & 2,14 & $3 \mathrm{I}, 7$ \\
\hline - & 一 & 55 à $65-\ldots \ldots \ldots \ldots \ldots$ & 619 & 16,2 & 2,25 & $3^{6,4}$ \\
\hline 一 & - & $65 \grave{a} 75-\ldots \ldots \ldots \ldots \ldots$ & 612 & 16,3 & 2,42 & 39,5 \\
\hline$\rightarrow$ & - & 75 à $85-\ldots \ldots \ldots \ldots \ldots$ & 667 & 15,0 & 2,84 & 42,5 \\
\hline - & - & 85 à $95-\ldots \ldots \ldots \ldots \ldots$ & $64 \mathrm{I}$ & 15,6 & 2,89 & 46,5 \\
\hline & & sommation moyenne pour $\mathrm{i} k$ & Ao cri & $\begin{array}{l}\text { I } 30,3 \\
\text { u.f. }\end{array}$ & & 267,4 \\
\hline
\end{tabular}

\section{2. - Conditions les plus défavorables}

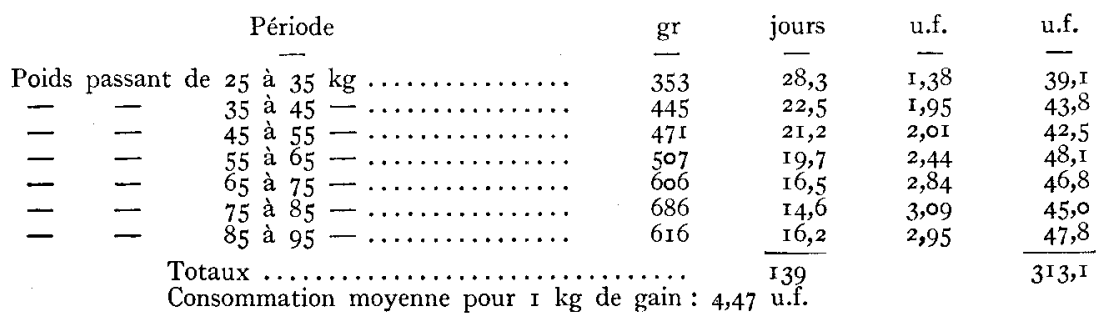


On voit ainsi qu'il existe une différence considérable entre la consommation qu'il serait possible d'obtenir dans les meilleures conditions $-3,82$ u. f. par $\mathrm{kg}$ - et celle qu'un aménagement particulièrement défectueux des porcheries pourrait théoriquement entraîner - 4,47 u. f. par $\mathrm{kg}$. - Le coût des aliments nécessaires à l'engraissement d'un porc serait dans ce dernier cas supérieur de $\mathrm{I} 7 \%$ à celui du premier.

Il convient de tenir compte de ces faits pour interpréter convenablement les résultats des épreuves de contrôle, car les animaux soumis à ces épreuves pendant la période d'hiver subissent un sévère handicap par rapport à ceux qui ont été engraissés au cours de la belle saison.

\section{CONCLUSION}

Les conditions dans lequelles vivent les porcs exercent une influence sur leur rapidité de croissance et sur l'indice de consommation exprimée en unités fourragères par $\mathrm{kg}$ de gain. Par rapport aux conditions moyennes de la Station de Bois-Corbon, des différences considérables s'observent entre les résultats obtenus au cours des différents mois de l'année ; les animaux s'engraissent avec une dépense moindre de nourriture pendant la période de juin à novembre que pendant la période de décembre à mai. Des écarts notables séparent à ce point de vue les observations faites sur des animaux de poids différents. L'influence de la saison semble plus importante sur la croissance des sujets de poids intermédiaire de 35 à $75 \mathrm{~kg}$ que sur les animaux plus jeunes ou sur les animaux parvenus à la dernière phase de leur engraissement. L'amélioration systématique du microclimat des porcheries pourrait exercer vraisemblablement une heureuse influence sur l'économie de la production du porc. 